\title{
A Comparison of intrathecal diamorphine with systemic analgesia following adolescent idiopathic scoliosis surgery
}

Dr R Krishnan, Dr T Ng, Dr A Strachan, Dr R Brinkler, Dr R Vijayraghavan

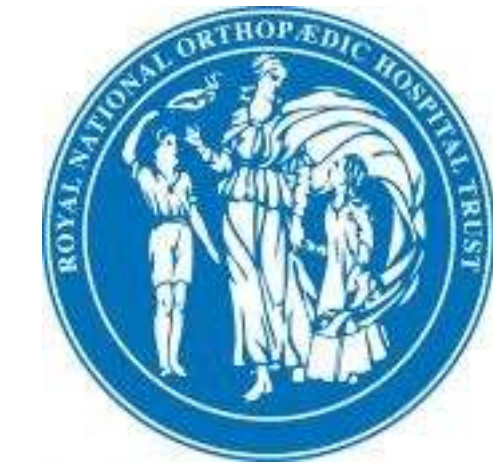

Royal National Orthopaedic Hospital, Stanmore

\section{Introduction}

Scoliosis surgery can result in severe post-operative pain. Currently post-operative pain management strategy consists of systemic opioids. High doses of opioids administered to achieve satisfactorily analgesia result in adverse effects such as nausea and vomiting, constipation, pruritus and respiratory depression.

Recently, alternative methods of delivering analgesia have been studied. Intrathecal morphine following spinal surgery is associated with an increased incidence of late respiratory depression. There are currently no studies that compare intrathecal diamorphine with systemic methods of analgesia following scoliosis surgery. The aim of our study is to compare the safety and efficacy of spinal diamorphine with systemic analgesia in patients undergoing adolescent idiopathic scoliosis surgery.

\section{Material and methods}

Study design

$>$ Retrospective comparative study

\section{Patient sample}

$>$ Sixty children under 18 years of age with adolescent idiopathic scoliosis undergoing posterior spinal fusion performed by 2 surgeons were included. $>$ Spinal diamorphine was injected by the anaesthetist with the patient under general anaesthesia before the surgical incision

\section{Outcome measures}

$>$ Postoperative analgesic requirement

$>$ Immediate recovery and postoperative pain scores

$>$ Estimated blood loss

$>$ Side effects

$>$ Critical care and hospital length of stay

$>$ Indicators of gastrointestinal function

\section{Results}

$>$ Mean dose of diamorphine injected intrathecally- $1.48 \mathrm{mg}$ (range 1-2 mg).

$>81 \%$ patients in the diamorphine group had mild pain $(<2 / 10$ of

\begin{tabular}{|l|l|l|l|l} 
Diamorphine & $\begin{array}{l}\text { Non } \\
\text { Diamorphine }\end{array}$ \\
\hline
\end{tabular}

Number of

30

30

patients

Age (years) $\quad 14.7$

15.6

visual analogue scale) in recovery compared to $7 \%$ in the diamorphine group

$>59 \%$ patients had no or mild pain up to 24 hours postop

Weight (kg) 57

compared to $6.7 \%$ in non diamorphine group

> Diamorphine group had less PCA opioid consumption (86.8 mg vs. $111 \mathrm{mg}$ )

Estimated $\quad 442$

413

$>$ The length of stay in critical care and hospital were similar in both groups

Blood Loss

Bowel

$>2$ patients in the diamorphine and 1 in the nondiamorphine group required naloxone bolus for respiratory depression opening (post op days) without airway intervention

$>$ Incidence of pruritis higher in diamorphine group (67\% vs. $33 \%)$

\section{Conclusions}

$>$ Spinal diamorphine provides better analgesia than conventional methods following lumbar spinal surgery. $>$ Spinal diamorphine is safe even in the dose of 20-30 micrograms/ $\mathrm{kg}$ as administered in this study. $>$ Randomised controlled trials are needed to find out the optimal dose of intrathecal diamorphine which would maximise the benefits without undue increase in side effects. 